\title{
A first principles technique for the analysis of alloy phase stability in random binary alloys
}

\author{
A MOOKERJEE* \\ S.N. Bose National Centre for Basic Sciences, DB17, Sector 1, Salt Lake City, Calcutta \\ 700064 , India \\ Abstract. In this communication we introduce the augmented-space recursion method \\ coupled with the orbital peeling technique, as a powerful tool for the calculation of effective \\ cluster interactions, useful for the study of alloy phase stability. An application to the well \\ studied PdV system has been carried out.
}

Keywords. First principles technique; random binary alloys.

\section{Introduction}

A bulk of the study of alloy phase diagrams have been the setting up and understanding of phenomenological models. The purpose of the models, distilled from extensive experimental data and physical insight, is to classify data using physical parameters felt to be crucial in the determination of phase stability and then by extrapolation (or interpolation) to make predictions. The idea is to use the chosen physical parameters as coordinates in a parameter space. It is then hoped that physical systems which are in the neighbourhood of one another should display similar features. By continuity, the parameter space is divided into non-overlapping domains of stability. Types and numbers of coordinates used is extensive and the reader is referred to several excellent reviews (Pearson 1972; Girgis 1983; Pettifor 1988; Villars et al 1989). Some of the popular parameters are those related to size effects and electronegativity, average electronic concentrations, covalency and ionicity (Phillips), valence electron concentrations (Hume-Rothery) or the Mendeleev number (Pettifor).

In spite of their frequent success and physical insight into ordering that these phenomenological laws often provide, non-critical and immoderate use of these rules often lead to serious disappointments. The geometric language suggested by the rules can be misleading. It is not clear how to define a metric on the parameter space, so that the concept of a neighbourhood is not at all clear. Also it is not clear whether the chosen coordinates are independent of each other or indeed, at all relevant. For example, size effects and electron concentrations can be related directly to each other in a class of materials but not in another. Each class then has its own rules and a comprehensive understanding is lacking.

It has therefore become clear that a first principles microscopic understanding of phase stability is important.

In recent years, there has been considerable interest in the first principles calculation of alloy ordering tendencies at $T=0^{\circ} \mathrm{K}$. In order to understand the onset of ordering in

\footnotetext{
* This work was done in collaboration with Tanusri Saha and Indra Dasgupta, S N Bose National Centre for Basic Sciences, Calcutta
} 
random alloys, one needs a derivation of the lowest configurational energy for a specified alloy system. Models have been formulated to represent the configurational energies in terms of effective multisite interactions, in particular, effective pair interactions (Gonis et al 1987). Within this approach, the analysis of alloy ordering tendencies and phase stability reduces to the accurate and reliable determination of effective pair interactions. Traditionally there have been two different approaches of obtaining the effective pair interactions. The first approach is to start with the electronic structure calculation and total energy determination of ordered super-structures of the alloy and to invert these total energies to get the effective pair potentials by the ConnollyWilliams method (Connolly and Williams 1983). The other approach is to start with the disordered phase, set up a perturbation in the form of concentration fluctuations associated with an ordered phase and study whether the alloy can sustain such a perturbation. This approach includes the generalized perturbation method (GPM) (Ducastelle and Gautier 1976), the embedded cluster method (ECM) (Gonis and Garland 1977) and the concentration wave approach (Gyorffy and Stocks 1983). Most of the work on the electronic structure of the disordered alloys has been based so far on the coherent potential approximation (CPA). The CPA being a single-site approximation has its own limitations. In addition, self-consistency involved in it invokes subtle convergence procedures at each energy point.

Analytic generalizations beyond the single site CPA, like the cluster CPA (Kumar et al 1982) based on the augmented space formalism and the travelling cluster approximation (TCA) (Mills and Ratnavararaksa 1974) are beset with computational difficulties when cluster sizes are large. Garkusha et al (1990) and Lós and Repetsky (1994) proposed a cluster expansion of the multiple scattering $t$-matrix. However, the analyticity preserving property of the truncated expansion has not been established as yet. In a separate attempt to go beyond the single site approximation, de Fontaine and his group followed a different approach of direct configurational averaging (DCA) (Dreyssé et al 1989; Wolverton et al 1993), without resorting to any kind of single-site approximation. The effective pair and multisite interactions were calculated directly in real space for given configurations and the averaging was done, brute force, as it were, by summing over different configurations.

The augmented space formalism (ASF) put forward by one of us (Mookerjee 1973) provides a convenient means of configuration averaging which is not restricted by the above limitations. In a recent communication (Saha et al 1994) we have shown that the ASF coupled with the recursion technique of Haydock et al (1972) successfully reproduces the electronic density of states of random alloys without resorting to single site approximations and does not require the self-consistency loops of CPA and its generalizations. The aim of the present work is to employ this methodology to get effective pair interactions. Within the ASF, configuration averaging is done exactly, while the approximation within the subsequent recursion on augmented space can be carried out in a controlled manner and the estimates of errors produced have been carefully studied (Haydock 1988). One works directly in real space, augmented with the space of configurations and does away with the limitations of the $k$-space super cell methods. Since the pair interactions are differences in total energies of different pairs in the alloy medium, they can be obtained directly by the orbital peeling method, without going through large subtractive cancellation (Burke 1976). 


\section{The formalism}

\subsection{Effective pair interactions}

The starting point of the calculations is the expansion of the configuration energy in terms of effective cluster interactions. The expansion for the configuration energy $E$ for a binary alloy $A_{c} B_{1-c}$ may be written as:

$$
E\left(n_{i}\right)=E^{(0)}+\sum_{i=1}^{N} E_{i}^{(1)} \delta c_{i}+\sum_{i, j=1}^{N} E_{i j}^{(2)} \delta c_{i} \delta c_{j}+\cdots
$$

If $n_{i}$ are the occupation probabilities for the A type of atom, $\delta c_{i}$ the concentration fluctuation given by $n_{i}-c$, where $c=\left\langle n_{i}\right\rangle$. The coefficients $E^{(0)}, E^{(1)} \cdots$ are the effective renormalized cluster interactions (renormalized in the sense that contributions from self-retraced paths have been included). $E^{(0)}$ is the energy of the averaged disordered medium. $E_{i}^{(1)}$ is the interchange energy for species $A$ and $\mathrm{B}$, and it defines the single body interaction resulting from the interchange of a $\mathrm{B}$ atom with an $\mathrm{A}$ atom at site $i$ in the alloy. $E_{i j}^{(2)}$ is the effective renormalized pair interaction which is the difference in the single body interaction at $i$, when sites $j(\neq i)$ is occupied either by $\mathrm{A}$ or $\mathrm{B}$ atom.

The renormalized pair interactions express the correlation between two sites and are the most dominant quantities for the analysis of phase stability. We will retain terms up to pair interactions in the configuration energy expansion. Higher order interactions may usually be included for a more accurate and complete description.

At this point it is worth mentioning that our scheme of calculation of the renormalized pair interactions is similar to other methods based on embedding clusters in an effective medium. The calculation involves the determination of the electronic structure as well as averaging over different configurations of the system. It is precisely in this averaging scheme that the different methods based on the embedding method differ from one another. In the CPA-ECM the averaging is done within the framework of the CPA. In the DCA the averaging is done directly by summing over different random configurations. If the configurations are consistent with a particular concentration alone the method is called the canonical DCA (Berera 1990). If averaging is done over all configurations as well as all possible concentrations, the method is called the grand canonical DCA (Asta et al 1991). In our scheme we employ the augmented space recursion for carrying out configuration averaging.

The other commonly used approach for obtaining the effective cluster interactions is the GPM. The equivalence of the CPA-ECM and the properly renormalized GPM (which is a CPA based technique) has been established previously and, in fact, the CPA-ECM gives the exact summation of the partially renormalized GPM to all orders (Gonis et al 1987). A detailed comparison of the various methods will be made later.

The total energy of a solid may be separated into two terms: a one-electron band contribution $E_{\mathrm{BS}}$ and an electrostatic term $E_{\mathrm{ES}}$ which includes several contributions: the Coulomb repulsion of the ion cores, the correction for double counting terms due to electron-electron interaction in $E_{\mathrm{BS}}$ and a Madelung energy in case the solid has ionic character. The renormalized cluster interactions defined in (1) should, in principle, include both $E_{\mathrm{BS}}$ and $E_{\mathrm{ES}}$ contributions. Since the renormalized cluster interactions involve the difference of cluster energies, it is usually assumed that the electrostatic terms cancel out and only the band structure contribution is important. Obviously, 
such an assumption is not rigorously true, but it has been shown to be approximately valid in a number of alloy systems (including PdV which we study here) (Heine 1988). Most of the works where calculations start from the disordered side are based on the band structure contribution alone. Our work also has that assumption.

The effective pair interactions can be related to the change in the configuration averaged local density of states:

$$
E_{i j}^{(2)}=\int_{-\infty}^{E_{\mathrm{F}}} \mathrm{d} E\left(E-E_{\mathrm{F}}\right) \Delta n(E),
$$

where $\Delta n(E)$ is given by:

$$
\Delta n(E)=\sum_{I J \in \text { all pairs }} \operatorname{Im}\left\langle\operatorname{Tr}\left(E \mathbf{I}-\mathbf{H}^{I J}\right)^{-1}\right\rangle \xi_{I J}
$$

$\xi_{I J}$ is \pm 1 according to whether $I=J$ or $I \neq J$ and $\langle\cdots\rangle$ refer to configuration averaging. There are four possible pairs $I J$, which can be $\mathrm{AA}, \mathrm{AB}, \mathrm{BA}$ and $\mathrm{BB} . H^{I J}$ is the Hamiltonian of a system where all sites except $i$ and $j$ are randomly occupied, whereas the sites $i$ and $j$ are occupied by atoms of the type $I$ and $J$. This change in the averaged local density of states can be related to the generalized phase shift $\eta(E)$ through the equation:

$$
\Delta n(E)=\frac{\mathrm{d} \eta(E)}{\mathrm{d} E}
$$

where

$$
\eta(E)=\log \frac{\operatorname{det}\left\langle G^{\mathrm{AA}}\right\rangle \operatorname{det}\left\langle G^{\mathrm{BB}}\right\rangle}{\operatorname{det}\left\langle G^{\mathrm{AB}}\right\rangle \operatorname{det}\left\langle G^{\mathrm{BA}}\right\rangle}
$$

$G^{I J}$ is the resolvent of the hamiltonian $H^{I J}$. The generalized phase shift $\eta(E)$ can easily be calculated following the orbital peeling method of Burke (1976). The details of the orbital peeling method in the context of pair interaction has been mentioned in detail elsewhere (Dreyssé et al 1989; Wolverton et al 1993), and here we shall quote only the final result:

$$
\begin{aligned}
E_{i j}^{(2)} & =\sum_{I J \in a l l ~ p a i r s} \sum_{\alpha=1}^{9} \xi_{I J} \int_{-\infty}^{E_{\mathrm{F}}}\left(E-E_{\mathrm{F}}\right) \log \left\langle G_{\alpha}^{I J}\right\rangle, \\
& =\sum_{I J \in a l l ~ p a i r s} \sum_{\alpha=1}^{9}\left[\sum_{k=1}^{p-1} Z_{k}^{\alpha, I J}-\sum_{k=1}^{p} P_{k}^{\alpha, I J}+\left(N_{\mathrm{P}}^{\alpha, I J}-N_{\mathrm{Z}}^{\alpha, I J}\right) E_{\mathrm{F}}\right],
\end{aligned}
$$

where $G_{\alpha}^{I J}$ denotes the Green function corresponding to the Hamiltonian, where two atoms are embedded at sites $I$ and $J$ in which the orbitals from 1 to $(\alpha-1)$ are deleted at the site I. $Z_{k}^{\alpha, I J}$ and $P_{k}^{\alpha, I J}$ are the zeros and poles of $G_{\alpha}^{I J} \cdot N_{\mathrm{Z}}^{\alpha, I J}$ and $N_{\mathrm{P}}^{\alpha, I J}$ are the number of such zeros and poles below $E_{\mathrm{F}}$. This method of zeros and poles enables one to carry out the integration easily avoiding the multivaluedness of the integrand involved in the evaluation of the integral by parts. These zeros and poles are estimated from the recursion coefficients for $\left\langle G_{\alpha}^{I J}\right\rangle$. 


\subsection{Augmented space recursion}

As discussed in the previous section, the calculation of the effective pair interaction in our formalism reduces to the determination of the configuration averaged green functions $\left\langle G^{\mathrm{AA}}\right\rangle,\left\langle G^{\mathrm{BB}}\right\rangle,\left\langle G^{\mathrm{AB}}\right\rangle$ and $\left\langle G^{\mathrm{BA}}\right\rangle$. We shall employ the augmented space recursion coupled with the linearized tight-binding muffin tin orbital method (TBLMTO) introduced by Andersen and Jepsen (1984) for a first principle determination of these configuration averaged quantities. We shall take the most localized, sparse tight binding Hamiltonian derived systematically from the LMTO-ASA theory and generalized to random alloys, given by:

$$
\begin{aligned}
H_{i L, j L^{\prime}}^{\beta} & =C_{i L^{\prime}}^{\prime} \delta_{i L} \delta_{j L^{\prime}}+\Delta_{i L^{1 / 2}}^{1 / \beta} S_{i L, j L^{\prime}} \Delta_{j L^{\prime}}^{1 / 2}, \\
C_{i L}^{\prime} & =C_{L}^{\mathrm{A}} n_{i}+C_{L}^{\mathrm{B}}\left(1-n_{i}\right), \\
\Delta_{L}^{1 / 2} & =\left(\Delta_{L}^{1 / 2}\right)^{\mathrm{A}} n_{i}+\left(\Delta_{L}^{1 / 2}\right)^{\mathbf{B}}\left(1-n_{i}\right) .
\end{aligned}
$$

Here $i, j$ denote the lattice sites and $L=(l m)$ are the orbital indices (for transition metal $l \leqslant 2$ ). $C_{L}^{\mathrm{A}}, C_{L}^{\mathrm{B}}, \Delta_{L}^{\mathrm{A}}$ and $\Delta_{L}^{\mathrm{B}}$ are the potential parameters of the constituents $\mathrm{A}$ and $\mathrm{B}$ of the alloy, $n_{i}$ are the local site-occupation variables which randomly take values 0 or 1 according to whether the site is occupied by an A atom or not. The screened or tight binding structure function $S^{\beta}$ contains all the information on lattice geometry, and it is expressed in terms of conventional structure function $S^{0}$ and the screening parameter $\beta$ as

$$
S^{\beta}=S^{0}\left(1+\beta S^{\beta}\right)
$$

The Hamiltonian described by (7) is related to the nearly orthonormal $\gamma$-representation by the relation

$$
H^{\gamma}=E_{v}+h^{\gamma}=E_{v}+h^{\beta}-h^{\beta} o^{\beta} h^{\beta}+\ldots
$$

This ASA form of the orthonormalized Hamiltonian was used in the present work. Usually the expansion is truncated after the second term which is accurate to first order in $\left(E-E_{v}\right)$. The third term is necessary for systems with wide bands specially for $s, p$ states. We have used both first and second order approximations to ensure convergence. The configuration averaged green functions $\left\langle G_{\alpha}^{I J}(z)\right\rangle$ is $\left\langle\left(z I-H^{I J}\right)^{-1}\right\rangle$. Assuming that we embed $I$ and $J$ in sites 1 and 2 the Hamiltonian $H_{\alpha}^{I J}$ has the following form:

$$
\begin{aligned}
H_{\alpha}^{I J} & =\sum_{l=\alpha}^{9} C_{1, l}^{I} a_{1}^{\dagger} a_{1}+\sum_{l=1}^{9} C_{2, l}^{J} a_{2}^{\dagger} a_{2}+\ldots \\
& +\sum_{p \neq 1,2} \sum_{l=1}^{9}\left(C_{p, l}^{\mathrm{B}}+\delta C_{l} n_{p}\right) a_{p}^{\dagger} a_{p}+\ldots \\
& +\sum_{p \neq 1} \sum_{l=\alpha}^{9} \sum_{l^{\prime}=1}^{9} \Delta_{1, l}^{1 / 2, l} S_{l, l^{\prime}}^{1, p}\left(\Delta_{p, l^{\prime}}^{1 / 2, \mathrm{~B}}+\delta \Delta_{l^{\prime}}^{1 / 2} n_{p}\right) a_{1}^{\dagger} a_{p} \ldots \\
& +\sum_{p \neq 2} \sum_{l=1}^{9} \sum_{l^{\prime}=1}^{9} \Delta_{2, l}^{1 / 2, I} S_{l, l^{\prime}}^{2, p}\left(\Delta_{p, l^{\prime}}^{1 / 2, \mathrm{~B}}+\delta \Delta_{l^{\prime}}^{1 / 2} n_{p}\right) a_{2}^{\dagger} a_{p} \ldots \\
& +\sum_{p \neq 1} \sum_{l=1}^{9} \sum_{l^{\prime}=\alpha}^{9}\left(\Delta_{p, l}^{1 / 2, \mathrm{~B}}+\delta \Delta_{l}^{1 / 2} n_{p}\right) S_{l, l^{\prime}}^{p, 1} \Delta_{l, l^{\prime}}^{1 / 2, I} \Delta_{1, l^{\prime}}^{1 / 2, l} a_{p}^{\dagger} a_{1} \ldots
\end{aligned}
$$




$$
\begin{aligned}
& +\sum_{p \neq 2} \sum_{l=1}^{9} \sum_{l^{\prime}=1}^{9}\left(\Delta_{p, l}^{1 / 2, \mathrm{~B}}+\delta \Delta_{l}^{1 / 2} n_{p}\right) S_{l, l^{\prime}}^{p, 2} \Delta_{2, l^{\prime}}^{1 / 2, I} a_{p}^{\dagger} a_{2} \ldots \\
& +\sum_{p \neq 1,2} \sum_{q \neq 1,2} \sum_{l=1}^{9} \sum_{l^{\prime}=1}^{9}\left(\Delta_{p, l}^{1 / 2, \mathrm{~B}}+\delta \Delta_{l}^{1 / 2} n_{p}\right) S_{l, l^{\prime}}^{p, q}\left(\Delta_{q, l^{\prime}}^{1 / 2, \mathrm{~B}}+\delta \Delta_{l^{\prime}}^{1 / 2} n_{q}\right) \\
& \quad\left(a_{p}^{\dagger} a_{q}+a_{q}^{\dagger} a_{p}\right) .
\end{aligned}
$$

We note that the Hamiltonian has both diagonal as well as off diagonal disorder. We will retain this form of the Hamiltonian, and will not resort to any transformation as is done in single site approximations (Kudrnovský and Drchal 1990). In order to evaluate the configuration average we will employ the ideas of the ASF which puts configuration averaging on the same footing as quantum mechanical averaging by augmenting the hilbert space spanned by the wave functions with a configuration space spanned by different realizations of the random variables associated with the Hamiltonian.

Let us suppose that the Hamiltonian describing the system is characterized by a set of random occupation variables $\left\{n_{i}\right\}$, which are independent. The probability density of the variables $n_{i}$ is assumed io have finite moments of all orders, so that we may write

$$
p\left(n_{i}\right)=\frac{1}{\pi}\left\langle\gamma_{0}^{i}\left|\left(\left(n_{i}-i 0\right) \mathbf{I}-\mathbf{M}^{i}\right)^{-1}\right| \gamma_{0}^{i}\right\rangle
$$

where $\mathbf{M}^{i}$ is an operator defined on the configuration space $\phi^{i}$ of rank $N$, spanned by $N$ possible realizations of $n_{i}$. The augmented space theorem (Mookerjee 1973) now states that the configuration average of the resolvent $G\left(E,\left\{n_{i}\right\}\right)$ may be written as

$$
\left\langle G\left(E,\left\{n_{i}\right\}\right)\right\rangle=\left\langle F\left|z \tilde{I}-\tilde{H}\left(\left\{\tilde{M}^{i}\right\}\right)^{-1}\right| F\right\rangle,
$$

$\tilde{H}\left(\left\{\tilde{M}^{i}\right\}\right)$ is the same operator function in the augmented space of $\tilde{M}^{i}$ as $H\left(\left\{n_{i}\right\}\right)$ was of $n_{i}$ and $|F\rangle=\Pi^{\otimes}\left|\gamma_{0}^{i}\right\rangle$ is the configuration ground state in the augmented space. Thus the configuration averaging has been reduced to the problem of the ground state matrix element in the augmented space $\Psi=H \otimes \Phi$. For a system with $N$ sites and disorder described by binary probability distribution the rank of the space is $N \times 2^{N}$.

The construction of $\tilde{M}^{i}$ given the distribution of $n_{i}$ has been described in detail earlier (Mookerjee 1973). For a binary distribution $\tilde{M}^{i}$ is given by:

$$
\tilde{M}^{i}=x b_{i 0}^{\dagger} b_{i 0}+(1-x) b_{i 1}^{\dagger} b_{i 1}+\sqrt{x(1-x)}\left(b_{i 0}^{\dagger} b_{i 1}+b_{i 1}^{\dagger} b_{i 0}\right) .
$$

$\left(b_{i 0}^{\dagger}, b_{i 0}\right)$ and $\left(b_{i 1}^{\dagger}, b_{i 1}\right)$ are the creation and annihilation operators in the augmented space where each site is characterized by two states $(0,1)$, which may be identified with the up and down states of an Ising system. The configuration states may then be stored extremely efficiently in bits of words and the algebra of the Hamiltonian in the configuration space may mirror the multispin coding techniques used in numerical work with the Ising model.

Once we have identified the operators in the configuration space, it is worth while mentioning how one represents the basis in real and configuration space. A basis $|m\rangle$ in $H$ is represented by a column vector $C_{m}$ with zeros everywhere except at the $m$ th position. The inner product

$$
\begin{aligned}
\langle m|\odot| n\rangle & =C_{m}^{T} C_{n}, \\
a_{m}^{\dagger} a_{n} C_{p} & =\delta_{n p} C_{m} .
\end{aligned}
$$


A member of the basis in $\Pi_{i}^{\otimes} \phi_{i}$ has the form $\left|\gamma_{\lambda_{1}}^{1} \otimes \gamma_{\lambda_{2}}^{2} \ldots \otimes \gamma_{\lambda_{j}}^{j} \otimes \ldots\right\rangle$ where each $\lambda$ may be either 0 or 1 . In the usual terminology of ASF the number $C$ of 1 's defines the cardinality of the basis and the sequence of positions at which we have the 1 's $\left\{S_{\mathrm{C}}\right\}$ called the cardinality sequence labels the basis. Thus a binary sequence $B\left[C,\left\{S_{\mathrm{c}}\right\}\right]$ is a representation of the member of the basis in the configuration space. The dot product between these basis members is then

$$
B\left[C,\left\{S_{\mathrm{C}}\right\}\right] \odot B\left[C^{\prime},\left\{S_{\mathrm{C}^{\prime}}\right\}\right]=\delta_{\mathrm{CC}^{\prime}} \delta\left\{S_{\mathrm{C}} S_{\mathrm{C}^{\prime}}\right\} .
$$

Without going into the computational details we mention that the operations of $\tilde{H}$ on the configuration space amount to changing cardinally and cardinality sequences. Since the operations are restricted to the bits of words one can easily employ logical functions to define the operation of the Hamiltonian in the configuration space.

It is well known, that for a system described by a sparse Hamiltonian the recursion method is one of the widely used methods to generate a continued fraction expansion of the resolvent of the Hamiltonian. Haydock (1972) has mapped the contribution to the continued fraction coefficients to self-avoiding walks on the underlying space. He has shown that the dominant contribution comes from walks that wind round the initial starting state. This allows us to work only on a finite part of the augmented space. In practice the continued fraction is evaluated to a finite number of steps and then terminated by incorporating analyticity preserving terminators. Several terminators are available in the literature and we have chosen to use the terminator of Lucini and Nex (1987). The advantage of such a termination procedure is that the approximate resolvent retains the herglotz properties. It is interesting to compare this with the fact that in the cluster generalizations of the CPA one goes to great lengths to ensure herglotzicity and these approximations cannot maintain the accuracy in the band-widths. The terminator approximation preserves the first $2(N-2)$ moments of the density of states exactly. This represents the effect of a cluster at a distance $(N-2)$ from the starting state. It also maintains the correct band-widths, band-weights and the correct singularities at the band edges. If we carry out recursion on the augmented space, we can compute the configuration averaged green function directly. The method does not involve single site approximations and solution of self-consistent equations as required in the CPA and its generalizations. For non-isochoric alloys, the difference in atomic radii of the constituents lead to change in the electronic density of states, as confirmed by experiment (Wright et al 1987) and approximate theoretical techniques (Bose et al 1992). One thus expects that the mismatch of size produces, in addition to a relaxation energy $E_{R}$ contribution, a change in the band structure. Within our augmented space recursion (ASR), off-diagonal disorder in the structure matrix $S^{\beta}$ because of local lattice distortions due to size mismatch of the constituents, can be handled on the same footing as diagonal disorder in the potential parameters (Dasgupta et al 1994).

The augmented space recursion with the TB-LMTO Hamiltonian coupled with orbital peeling allows us to compute configuration averaged pair-potentials directly, without resorting to any direct averaging over several configurations. In an earlier communication (Saha et al 1994) we have discussed how one uses the local symmetries of the augmented space to reduce the Hamiltonian and carry out the recursion on a reducible subspace of much lower rank. If we fix the occupation of two sites, the local symmetry of the augmented space is lowered'(this is very similar to the lowering of spherical symmetry to cylindrical symmetry when a preferred direction is introduced in an isotropic system). We may then carry out the recursion in a suitably reduced subspace. 


\section{An illustrative example}

We shall now illustrate the usefulness of the formalism developed in the previous section in calculating the pair potentials for the fcc based ${P d_{c}}_{V_{1-c}}$ alloys for various values of concentration $c$. The calculation of the pair potential has been restricted up to fourth neighbour interactions. Our choice of the $\mathrm{Pd}-\mathrm{V}$ alloy is motivated by the fact, that in transition metal alloys (apart from few exceptions) arguments show that the most strongly ordered alloys will have an average band filling near the middle of the $d$-band, or somewhere near five $d$-electrons, whereas alloys with band filling close to completely empty or full $d$ level will tend towards phase separation. $\mathrm{Pd}-\mathrm{V}$ alloys, with constituents on opposite ends of the transition metal series, will order according to the above prescription. Further, stability properties of $\mathrm{Pd}-\mathrm{V}$ system is well studied both experimentally as well as by theoretical methods like KKR-CPA coupled with generalized perturbation method (Turchi et al 1988) and by LMTO coupled with direct configurational averaging (Dreyssé et al 1989; Wolverton et al 1993), so reliable results are available for comparison with our predictions.

We now mention briefly some details concerning the numerical part of the problem. Total energy density-functional calculations were performed for Pd and V in the fccstructure. The Kohn-Sham equations were solved in the local-density approximations (LDA). The LDA was treated within the context of the method of the linear muffin-tin orbitals (LMTO) in the atomic sphere approximation. The computations were performed semi-relativistically (including scalar relativistic corrections) and the exchangecorrelation potential of Von Barth and Hedin was used. The basis set was composed of $l=0,1,2$ orbitals, so that the occurring matrices were of order 9 . The potential parameters $\Delta_{l}^{\mathrm{I}}$ and $\gamma_{l}^{\mathrm{I}}$ of the constituent I were scaled by factors $\left(s^{\mathrm{I}} / \mathrm{s}^{\mathrm{alloy}}\right)^{2 l+1}$ to account for the fact that the Wigner-Seitz radius of constituent $I, s^{I}$ is different from that of the alloy, $s^{\text {alloy }}$. These potential parameters were used to parametrize the alloy Hamiltonian. This choice of different Wigner-Seitz radius for $\mathrm{Pd}$ and $\mathrm{V}$ allowed one to take into account charge self-consistency approximately, yet accurately and consistently, as emphasized by Kudrnovský et al (1990). For the purpose of augmented space recursion, a four shell augmented space map was generated from a cluster of 400 sites. We calculated the local density of states and fermi-energy with second order LMTO-ASA Hamiltonian through the recursion method exact up to eight levels of recursion and with analytic terminator of Lucini and Nex (1987).

Figure 1 shows the total density of states for $\mathrm{Pd}-\mathrm{V}$ alloy, for various concentrations with the positions of the fermi level shown. In order to maintain accuracy the Fermi energy $E_{\mathrm{F}}$ has been calculated from the second order Hamiltonian. For the calculation of the pair potential we followed the same methodology coupled with orbital peeling discussed in detail earlier with first order Hamiltonian. We found that since pair potentials are differences of integrated quantities, there seemed to be little appreciable change in doing the calculation with the second order Hamiltonian.

In figure 2 we have plotted the nearest neighbour effective pair interactions, as functions of (a) band filling and (b) energy, at three different concentrations for the Pd-V alloy. The relation between the two figures can be understood by looking at the variation of the number of states per spin with energy. This is shown in figure $2 \mathrm{c}$. The shape of the curves is in agreement with those obtained by other methodologies (Gonis et al 1987; Dreyssé et al 1989; Wolverton et al 1993), consisting of a phase separating region at the band edges and an ordering region near the centre. The magnitude of 


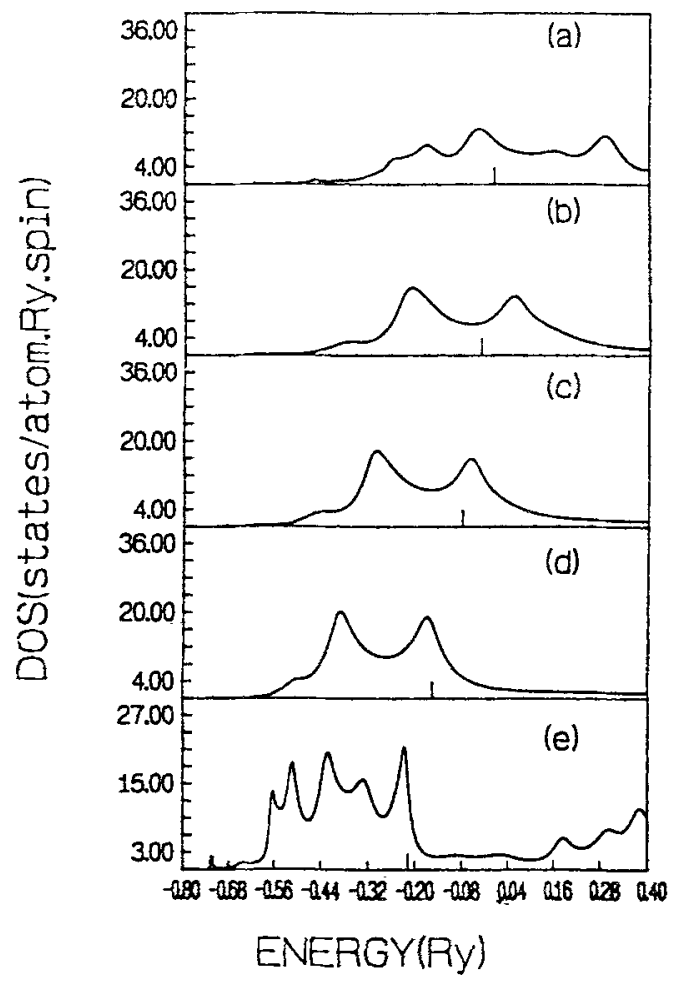

Figure 1. Density of states vs energy for $\mathbf{P d}_{c} V_{1-c}$ alloys. (a) $c=0 \cdot 0$, (b) $c=0 \cdot 25$, (c) $c=0 \cdot 5$, (d) $c=0.75$ and $(\mathbf{e}) c=1 \cdot 0$. The vertical lines mark the positions of the Fermi energies.

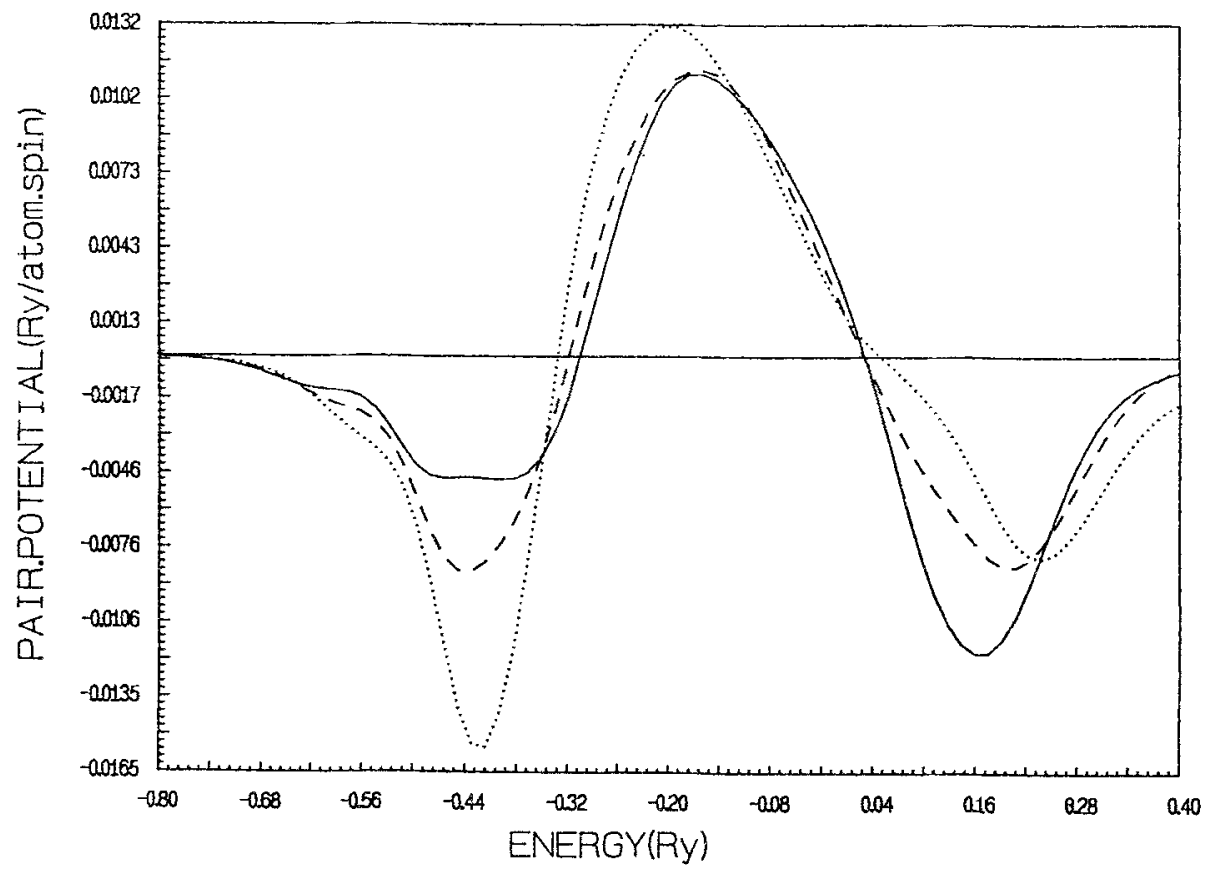

Figure 2. a. The nearest neighbour pair potential $V_{1}$ vs band filling for $\mathrm{Pd}_{c} \mathrm{~V}_{1-c}$ alloys: full curve, $c=0.5$; dashed curve, $c=0.75$; and dotted curve $c=0.25$. Vertical lines mark the band flling in the three different concentrations, $b$. the nearest neighbour pair potential $V_{1}$ ys energy for $\mathrm{Pd}_{c} \mathrm{~V}_{1-c}$ alloys: full curve, $c=0.5$; dashed curve, $c=0.75$; and dotted curve $c=0.25$. Vertical lines mark the Fermi energies and $c$. the number of states per atom as functions of energy for the three alloys mentioned in $\mathbf{2 a - b}$. The vertical lines mark the Fermi energies. 


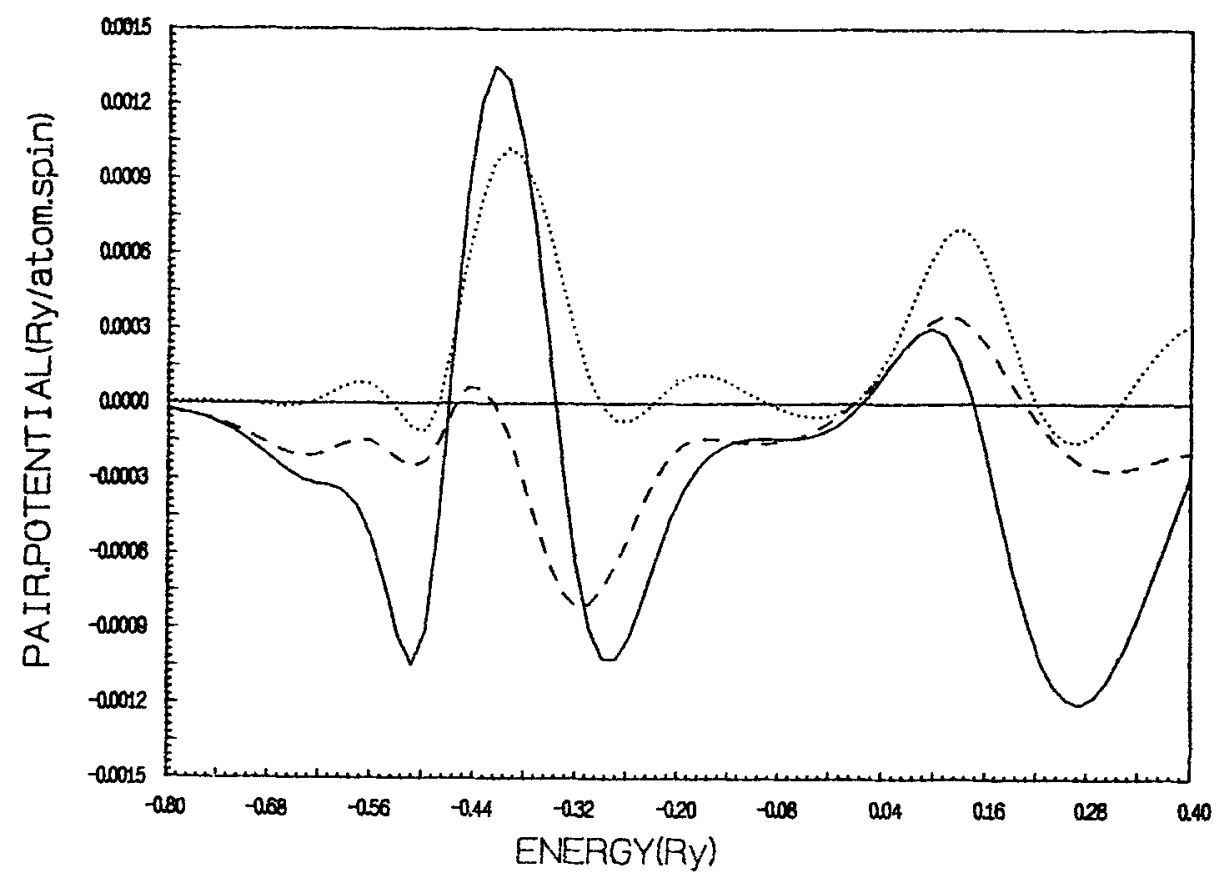

Figure 3. The $n$ neighbour pair potentials vs band filling for $c=0.5$, full curve, $n=2$; dashed curve, $n=3$ and dotted curve, $n=4$. Vertical lines mark the band filling in the three different concentrations.

the effective pair interaction decreases with distance with increasing number of nodes.

In figure 3 we have plotted $V_{1}, V_{2}, V_{3}$ and $V_{.4}^{*}$ for $c=0.5$ as a function of band filling to demonstrate this point. This in turn indicates the rapidly convergent properties of the configuration energy expanded in terms of effective cluster interactions. We find that $\left|V_{1}\right| \gg \mid V_{2}, V_{3}$ or $V_{4} \mid$ so that the ordered structures appear only when $V_{1}>0$.

Once we have obtained the effective pair interactions, they can be used to calculate the ordering energy, hence the relative stability of various ordered phases at a given concentration. At this point one can calculate the ordering energy expressed in terms of effective pair interactions for various stable phases at a given concentration to analyze the stability properties. However to discuss the stability properties of $\mathrm{Pd}-\mathrm{V}$ alloy we will follow the method of Kanamori and Kakehashi (1977) and calculate the antiphase boundary energy. Using the method of geometric inequalities these authors determined the ground state of $3 \mathrm{D}$ Ising model including pair interaction up to fourth NN interactions. They expressed the configurational energy as:

$$
E_{\mathrm{C}}=\sum_{k} V_{k} p_{k}
$$

where $p_{k}$ is the total number of $k$ th neighbouring BB pairs in a given configuration $C$. The antiphase boundary energy between two closely related ordered superstructure

$* V_{n}=E_{i j}^{2},\left|r_{i}-r_{j}\right|=n a$ where $a$ is the lattice constant. 
Table 1. Values of $p_{k}$ for various superstructures at concentrations $0-25$ and $0-5$.

\begin{tabular}{llllll}
\hline$k$ & 1 & 2 & 3 & 4 & $c$ \\
\hline $\mathrm{LI}_{2}$ & 0 & 3 & 0 & 6 & 0.25 \\
$\mathrm{DO}_{22}$ & 0 & 2 & 4 & 2 & 0.25 \\
$\mathrm{LI}_{0}$ & 2 & 3 & 4 & 6 & 0.5 \\
$\mathrm{~A}_{2} \mathrm{~B}_{2}$ & 2 & 2 & 8 & 2 & 0.5 \\
\hline
\end{tabular}

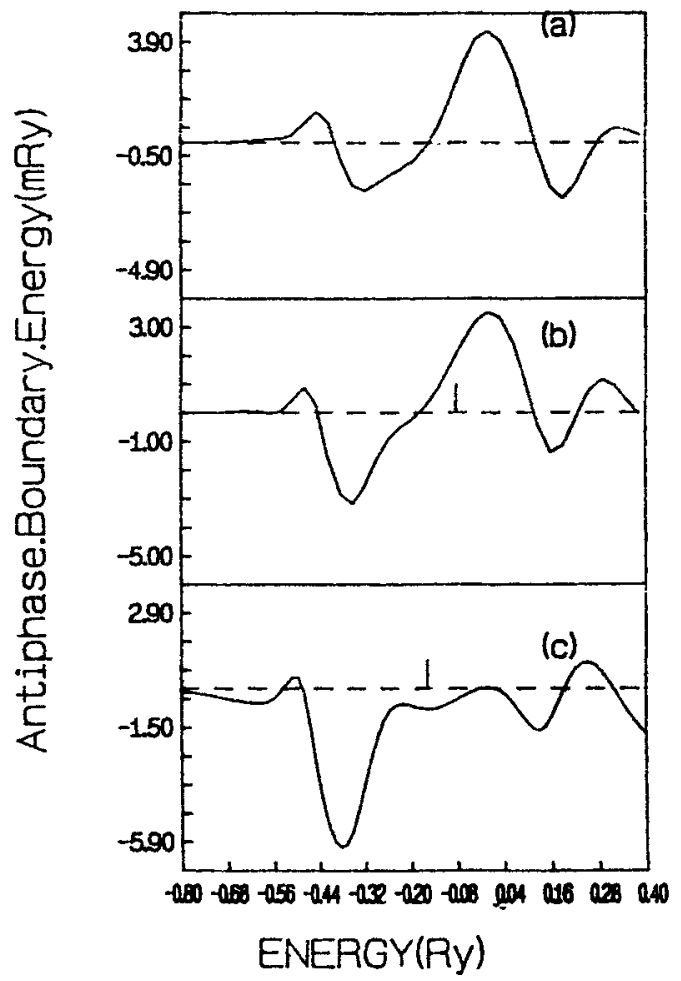

Figure 4. The antiphase boundary energies for $c=$ (a) 0.25 , (b) 0.5 and (c) 0.75 . Vertical lines mark the band filling in the three different concentrations.

configurations $C$ and $C^{\prime}$ is given by

$$
\varepsilon=E_{C}-E_{C^{\prime}}
$$

In table 1 we list the values of $p_{k} / N_{C}$ for different configurations.

In figure 4 we have plotted this anti-phase boundary energy

$$
\varepsilon=-V_{2}+4 V_{3}-4 V_{4}
$$

as a function of band filling for $\mathrm{Pd}-\mathrm{V}$ with $c=0.25,0.5$ and 0.75 . The number of zeros is in agreement with the arguments based on moments (there has to be at least four zeros). 
In the fcc lattice for $c=0.75$, the only possible ground states in the presence of interactions up to fourth nearest neighbours are $\mathrm{LI}_{2}$ and $\mathrm{DO}_{22}$. The two structures have identical numbers of $\mathrm{AB}$ nearest pairs, which means that the difference in energy is much smaller than $V_{1}$ and is given by the anti-phase boundary energy. It has been proved rigorously by Kanamori and Kakehashi (1977) from the ground state analysis of the $3 \mathrm{~d}$ Ising model Hamiltonian that $\mathrm{DO}_{22}$ structure is stable if $\varepsilon<0$ and $\mathrm{LI}_{2}$ structure is stable if $\varepsilon>0$. We find from figure 4 that $\varepsilon<0$ at $E=E_{\mathrm{F}}$ for $c=0.75$ suggesting that at this concentration $\mathrm{DO}_{22}$ structure is stable. A similar analysis shows that $\varepsilon>0$ for $c=0.25$ at $E=E_{\mathrm{F}}$ and here the $\mathrm{LI}_{2}$ structure is stable. This is further supported by the fact that an exchange of stability between $\mathrm{LI}_{2}$ and $\mathrm{DO}_{22}$ occurs for large electron number. For $c=0 \cdot 5$, the possible ground state configurations are $\mathrm{LI}_{0}$ and $A_{2} B_{2}$ and we find $L_{1}$ to be more stable by a similar analysis of anti-phase boundary energy in agreement with experiments and theoretical studies based on other methods.

In conclusion our results demonstrate that augmented space recursion and orbital peeling in conjunction with LMTO formalism, constitute a viable and computationally feasible approach to the calculation of phase stability in binary substitutionally disordered alloys. However, before accurate quantitative answers can be obtained, further work is necessary in order to incorporate self-consistency in the total energy, exchange and correlation effects, elastic interactions and lattice relaxation into theory of alloy phase stability. This methodology is capable of tackling these sophistications and the study still form an open problem.

\section{References}

Andersen O K and Jepsen O 1984 Phys. Rev. Lett. 532571

Asta M, Wolverton C, de Fontaine D and Dreyssé H 1991 Phys. Rev. B44 4907

Berera A 1990 Phys. Rev. B42 4311

Bose S K, Kudrnovský J, Jepsen O and Andersen O K 1992 Phys. Rev. B45 8272

Burke N R 1976 Surf. Sci. 58349

Connolly J W D and Williams A R 1983 Phys. Rev. B27 5169

Dasgupta I, Saha T and Mookerjec A 1994 Phys. Rev. B51

Dreyssé H, Berera A, Wills L. T and de Fontaine D 1989 Phys. Rev. B39 2442

Ducastelle F and Gautier F 1976 J. Phys. F6 2039

Garkusha V V, Loś V F and Repetsky S P 1990 Theor. Math. Phys. 8499

Girgis K 1983 Physical metallurgy (Amsterdam: North Holland) pp 219

Gonis A and Garland J W 1977 Phys. Rev. B16 2424

Gonis A, Zhang. X G, Freeman A J, Turchi P, Stocks G M and Nicholson D M 1987 Phys. Rev. B36 4630

Györffy B L and Stocks G M 1983 Phys. Rev. Lett. 50374

Haydock R 1972 Ph D Thesis, University of Cambridge, Cambridge

Haydock R 1988 Solid state physics (New York: Academic Press) 35

Haydock R, Heine V and Kelly M J 1972 J. Phys. C5 2845

Heine V 1988 Solid state physics (New York: Academic Press) 35

Kanamori J and Kakehashi Y 1977 J. Phys. (Paris) Colloq. $38 \mathrm{C} 7$

Kudrnovský J and Drchal V 1990 Phys. Rev. B41 7515

Kumar V, Mookerjee A and Srivastava V K 1982 J. Phys. C15 1939

Loś V F and Repetsky S P $1994 J$. Phys. Condens. Matter (in press)

Lucini M U and Nex C M M 1987 J. Phys. C20 3125

Mills R and Ratnavararaksa R 1974 Phys. Rev. B18 1200

Mookerjee A 1973 J. Phys. C6 1340

Pearson W B 1972 The crystal chemistry and physics of metals and alloys (New York: Wiley)

Pettifor D G 1988 Mater. Sci. Technol. 4675

Saha T, Dasgupta I and Mookerjee A 1994 J. Phys. Condens. Matter. 6 L245 
Turchi P E A, Stocks G M, Butler W H, Nicholson D M and Gonis A 1988 Phys. Rev. B37 5982

Villars P, Mathis K and Hulliger F 1989 The structure of binary compounds, cohesion and structure (Amsterdam: North Holland) 2 p. 1

Wolverton C, Ceder G, de Fontaine D and Dreyssé H 1993 Phys. Rev. B48 726

Wright H, Weightman P, Andrews P T, Folkerts W, Flipse C F J, Sawatsky G A, Norman D and Padmore H 1987 Phys. Rev. B35 519 